\title{
Nanoscale Bouligand Multilayers: Giant Circular Dichroism of Helical Assemblies of Plasmonic 1D Nano-Objects.
}

\author{
Hebing Hu, ${ }^{1 \ddagger}$ Sribharani Sekar, ${ }^{1 \ddagger}$ Wenbing Wu,${ }^{1}$ Yann Battie, ${ }^{2}$ Vincent Lemaire, ${ }^{1}$ \\ Oriol Arteaga, ${ }^{3,4}$ Lisa V. Poulikakos, ${ }^{5+}$ David J. Norris, ${ }^{5}$ Harald Giessen, ${ }^{6}$ \\ Gero Decher, ${ }^{* 1,} 7,8$ Matthias Pauly*1
}

1. Université de Strasbourg, CNRS, Institut Charles Sadron UPR22, 67000 Strasbourg, France

2. LCP-A2MC, Université de Lorraine, 57070 Metz, France

3. Dep. Física Aplicada, Feman Group, Universitat de Barcelona, Barcelona 08028, Spain

4. Institute of Nanoscience and Nanotechnology (IN2UB), Universitat de Barcelona, Barcelona 08028, Spain

5. Optical Materials Engineering Laboratory, Department of Mechanical and Process Engineering, ETH Zurich, 8092 Zurich, Switzerland

6. $4^{\text {th }}$ Physics Institute, University of Stuttgart, Pfaffenwaldring 57, Stuttgart 70569, Germany

7. International Center for Frontier Research in Chemistry, 67083 Strasbourg, France

8. International Center for Materials Nanoarchitectonics, Tsukuba, Ibaraki 305-0044, Japan

Current affiliation: ${ }^{\dagger}$ Department of Mechanical and Aerospace Engineering, University of California San Diego, 9500 Gilman Drive, La Jolla, California 92093, United States

Equal contribution: ${ }^{\ddagger}$ These authors contributed equally

Corresponding authors: decher@unistra.fr, matthias.pauly@ics-cnrs.unistra.fr 


\section{Modeling}

The Mueller matrix of nanostructures is simulated in transmission according to the Berreman transfer matrix formalism (BTM). ${ }^{1}$ Three different structures with 1, 2 and 3 layers of AgNWs deposited on a transparent silica substrate are considered. These structures, depicted in Figure S1, are composed of two building blocks: a layer of oriented AgNWs and an organic layer of $\mathrm{PEI} /(\mathrm{PSS} / \mathrm{PAH})_{5} / \mathrm{PSS} / \mathrm{PEI}$.

1-layer metasurface

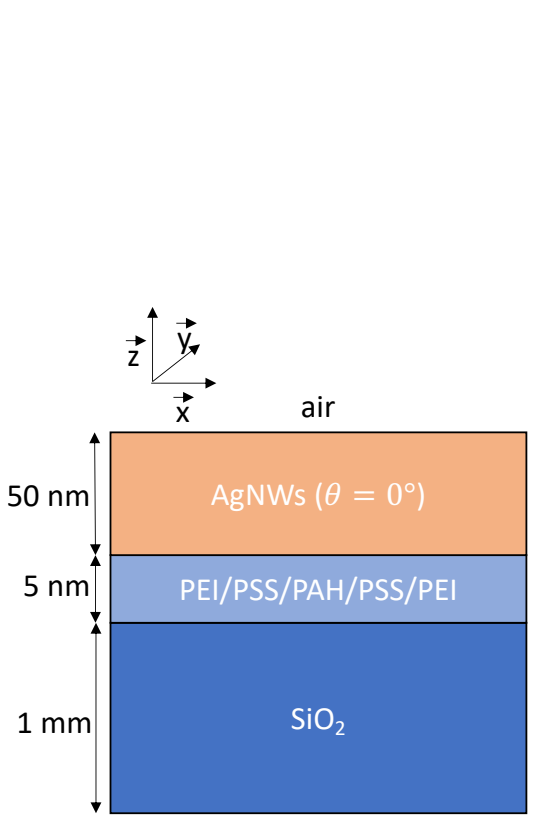

air 2-layer metasurface

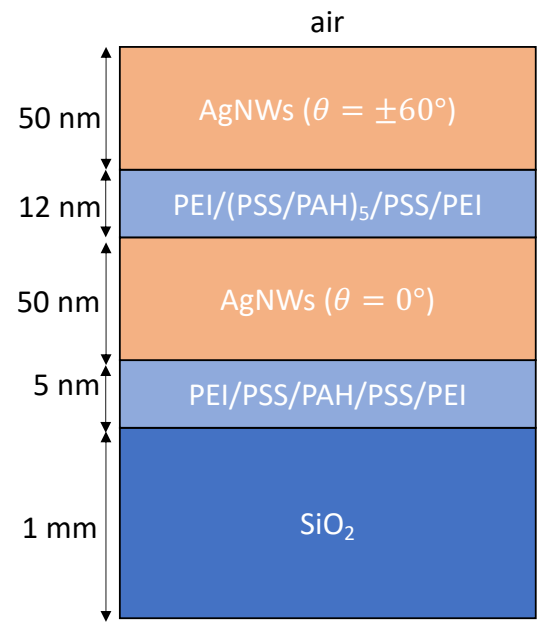

air 3-layer metasurface

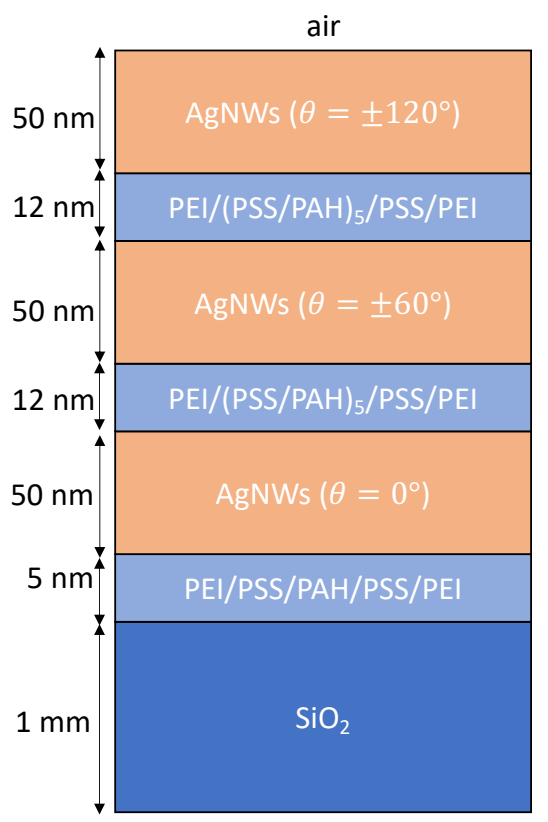

air

Figure S1: Structures of the 1, 2 and 3-layer metasurfaces used for the modeling. The optical axis of AgNW layer is in the $(\vec{x}, \vec{y})$ plane. $\theta$ is the angle between the optical axis and $\vec{x}$.

The BTM formalism requires the knowledge of the dielectric function of each layer. The dielectric functions of the organic layer and the layer of aligned AgNWs are deduced from ellipsometric measurements performed in reflection on films deposited on silicon substrate. The dielectric constant of the organic layers is set to 2.34. AgNW layers exhibit a uniaxial anisotropy with an optical axis parallel to AgNWs. The ordinary $\left(\varepsilon_{o}\right)$ and extraordinary $\left(\varepsilon_{e}\right)$ effective dielectric function of the aligned AgNW layer are shown in Figure S2. The ordinary effective dielectric function of AgNWs layer is dominated by a plasmon band located at $355 \mathrm{~nm}$. On the contrary, no plasmon band is observed in the extraordinary effective dielectric function. As $\varepsilon_{e} \varepsilon_{o}<0$, the AgNW layer is a hyperbolic metamaterial in the visible/NIR spectral range. 

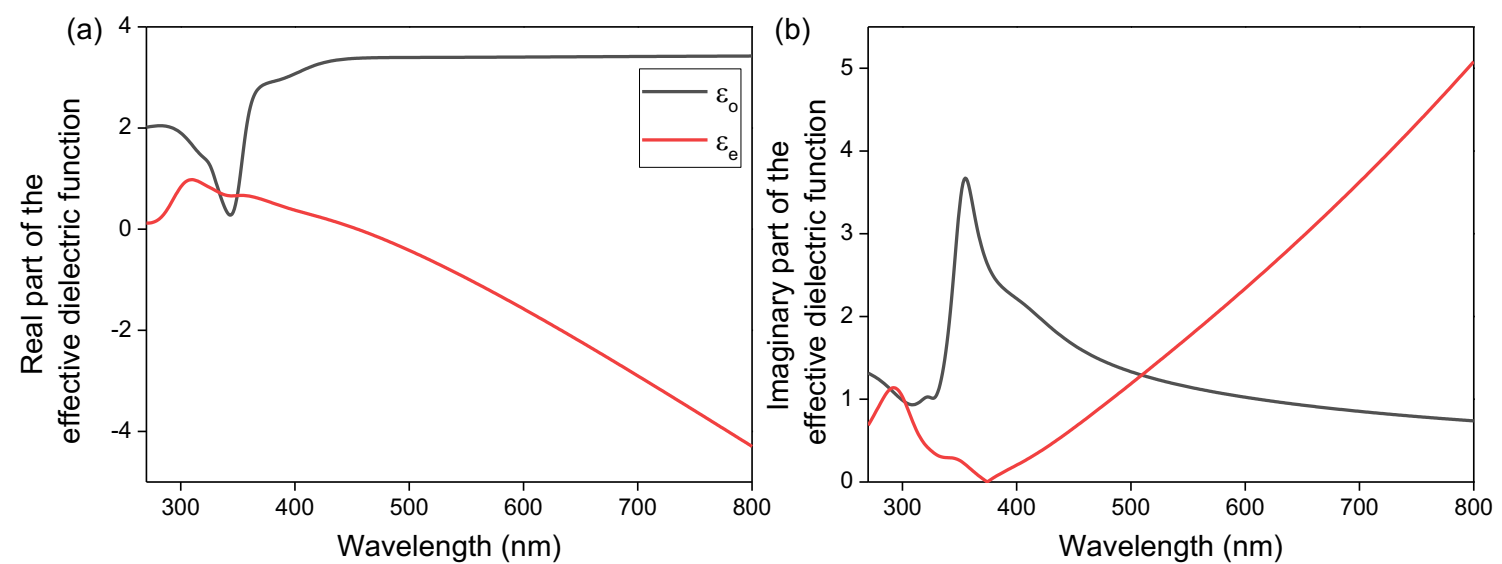

Figure S2: (a) real part and (b) imaginary part of the ordinary and extraordinary effective dielectric function of AgNW layer measured by ellipsometry in reflection mode.

The linear or circular dichroism and birefringence are then calculated from the Mueller matrix by using the differential Mueller matrix decomposition. ${ }^{2}$ 


\section{Supplementary figures}

a

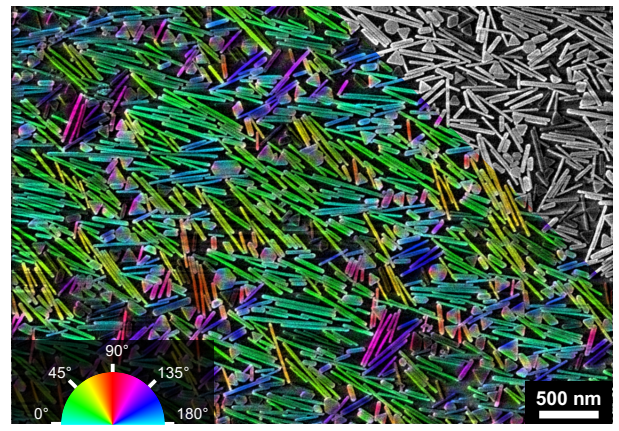

b

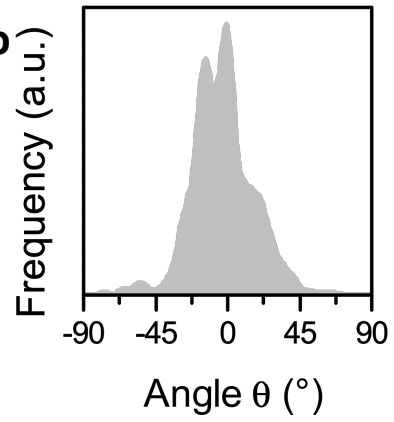

$S_{2 D}=\left\langle 2 \cos ^{2} \theta-1\right\rangle=0.45$

Figure S3: (a) SEM of a monolayer of oriented AuNRs color-coded according to their orientation and (b) angular distribution of the AuNR orientation.
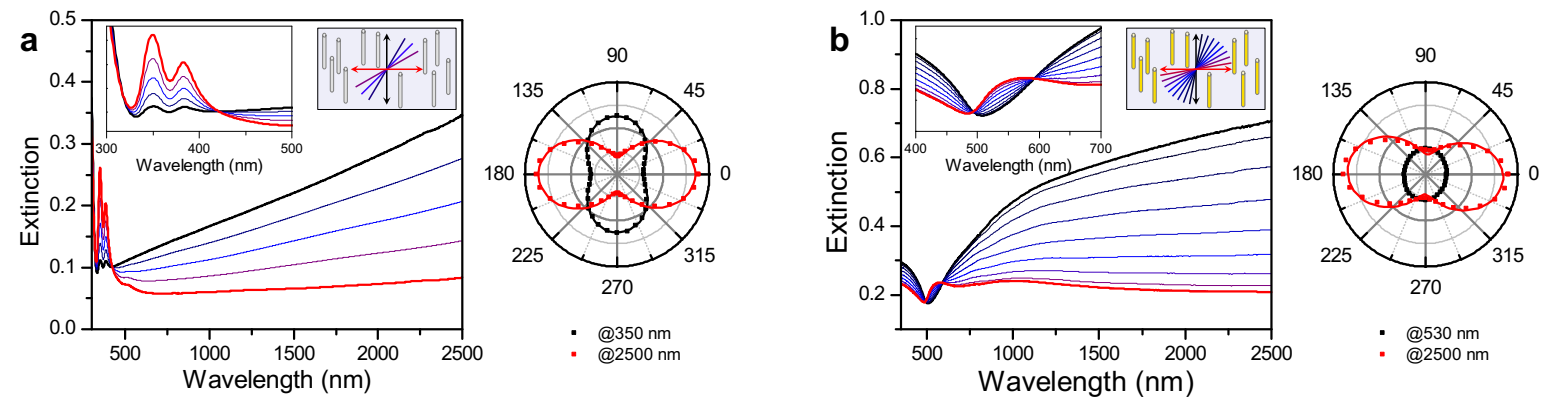

Figure S4: measured polarized extinction spectra of a monolayer thin film of (a) oriented AgNWs and (b) oriented AuNRs. The inset displays the direction of the linear polarization with respect to the orientation of the nanoobjects, and the spectra zoomed around the wavelength of the transverse LSPR modes. The polar plots show the extinction at the wavelength of the transverse LSPR (in black, at $350 \mathrm{~nm}$ and $530 \mathrm{~nm}$ for the AgNWs and AuNRs respectively) and the longitudinal LSPR (in red, at $2500 \mathrm{~nm}$ for AgNWs and AuNRs).
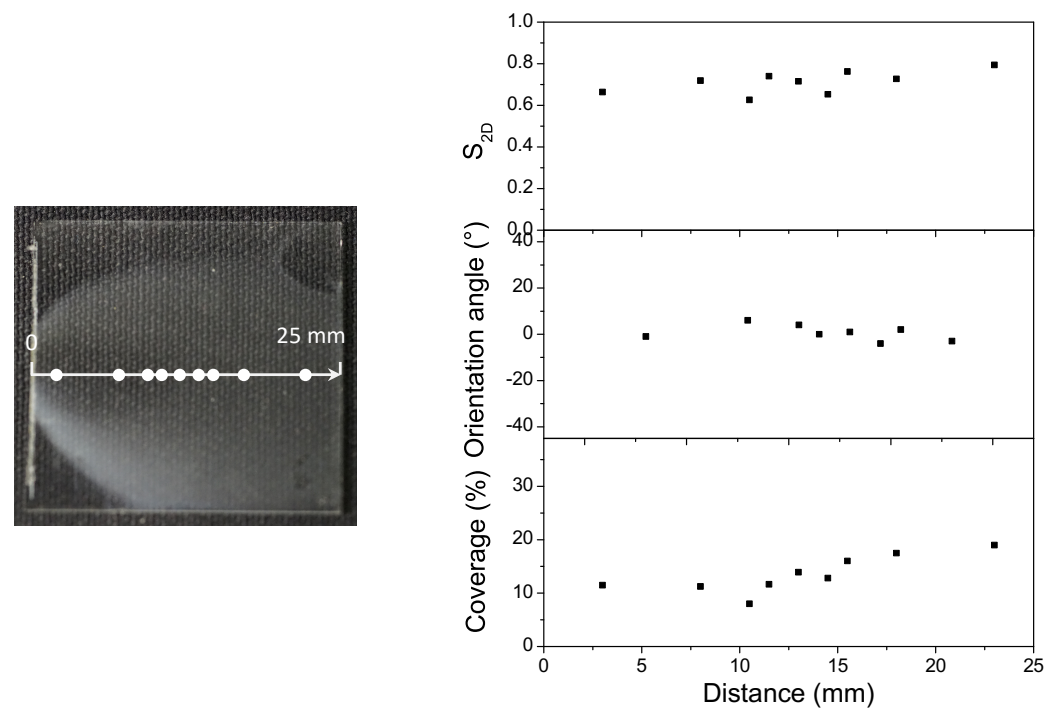

Figure S5: optical photograph of an AgNW oriented monolayer thin film deposited on a $25 \times 25 \mathrm{~mm}^{2}$ substrate. The coverage, average orientation angle and nematic order parameter $\mathrm{S}_{2 \mathrm{D}}$ have been measured on SEM images of the thin film taken at various positions along the central axis of the spray patter (as indicated by the white points on the image), highlighting the good homogeneity in terms of density of nanoobjects, orientation angle and order parameter. 
a

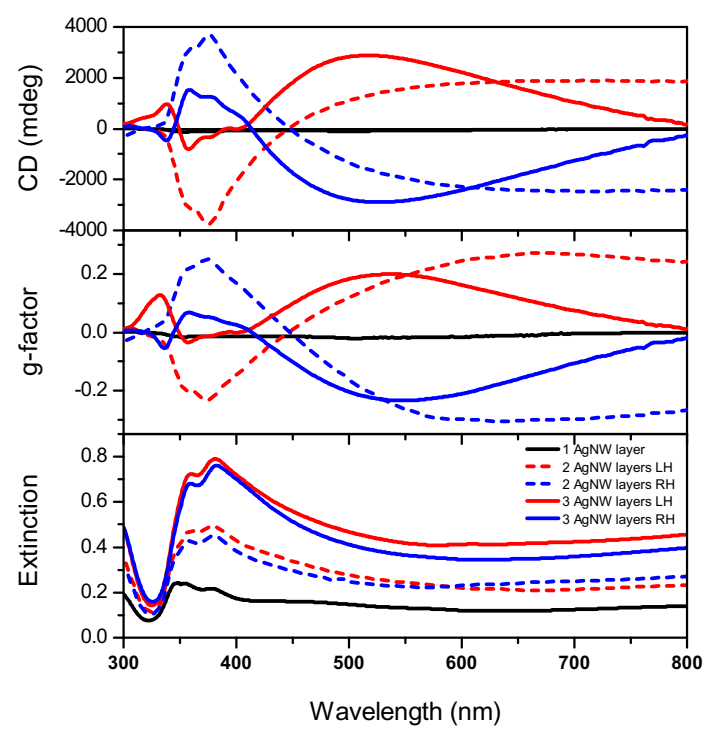

b

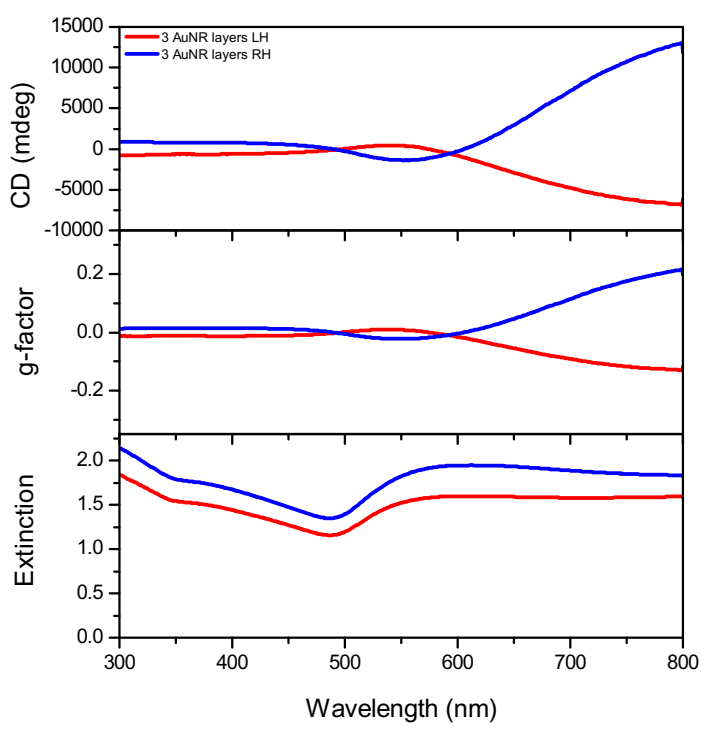

Figure S6: measured CD, g-factor and extinction spectra of (a) AgNW and (b) AuNR chiral metasurfaces. For the AgNW thin films, the spectra measured after deposition of the $1^{\text {st }}$ layer (black), $2^{\text {nd }}$ layer (dashed line) and $3^{\text {rd }}$ layer (full line) are given.
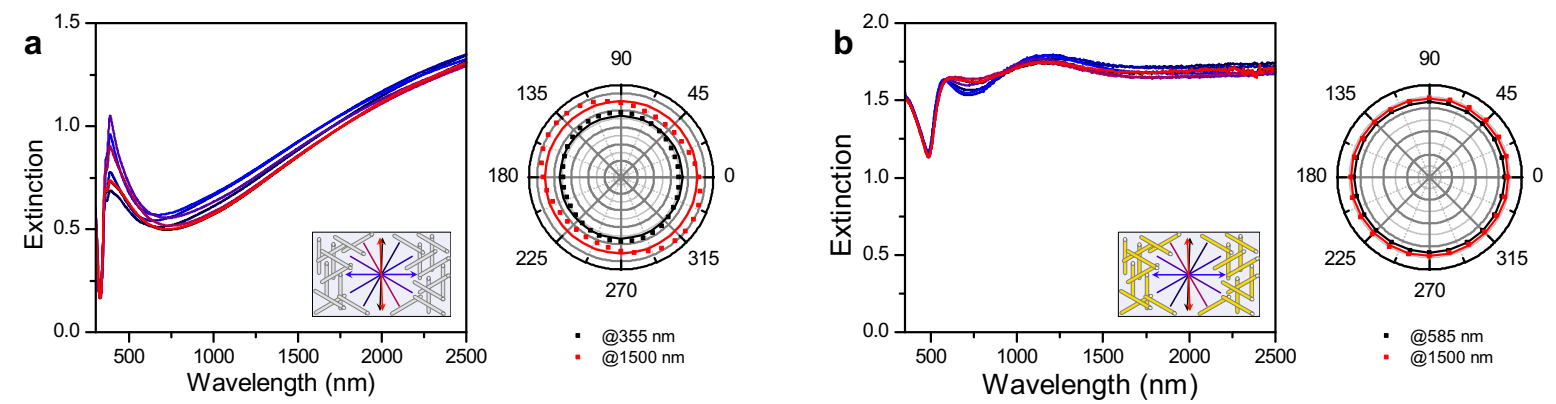

Figure S7: measured polarized extinction spectra of 3-layer chiral metasurfaces of (a) AgNWs and (b) AuNRs. The inset displays the direction of the linear polarization with respect to the sample. The polar plots show the extinction at the wavelength of the transverse LSPR (in black, at $355 \mathrm{~nm}$ and $585 \mathrm{~nm}$ for the AgNWs and AuNRs respectively) and the longitudinal LSPR (in red, at $1500 \mathrm{~nm}$ for AgNWs and AuNRs). 


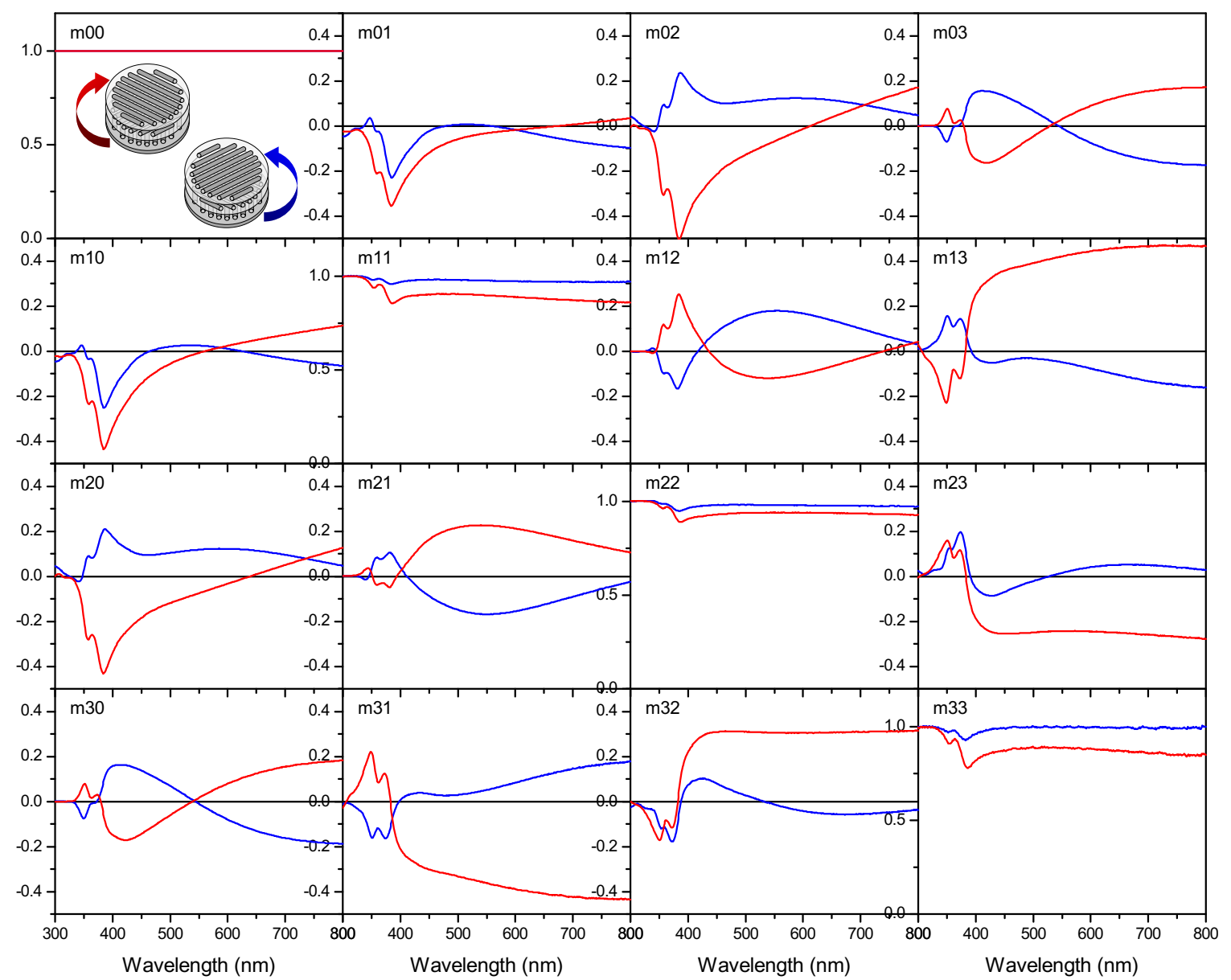

Figure S8: Mueller Matrix measured for a left- and right-handed 3-layer AgNW chiral metasurface. The AgNWs used here were synthesized according to the procedure described above. 


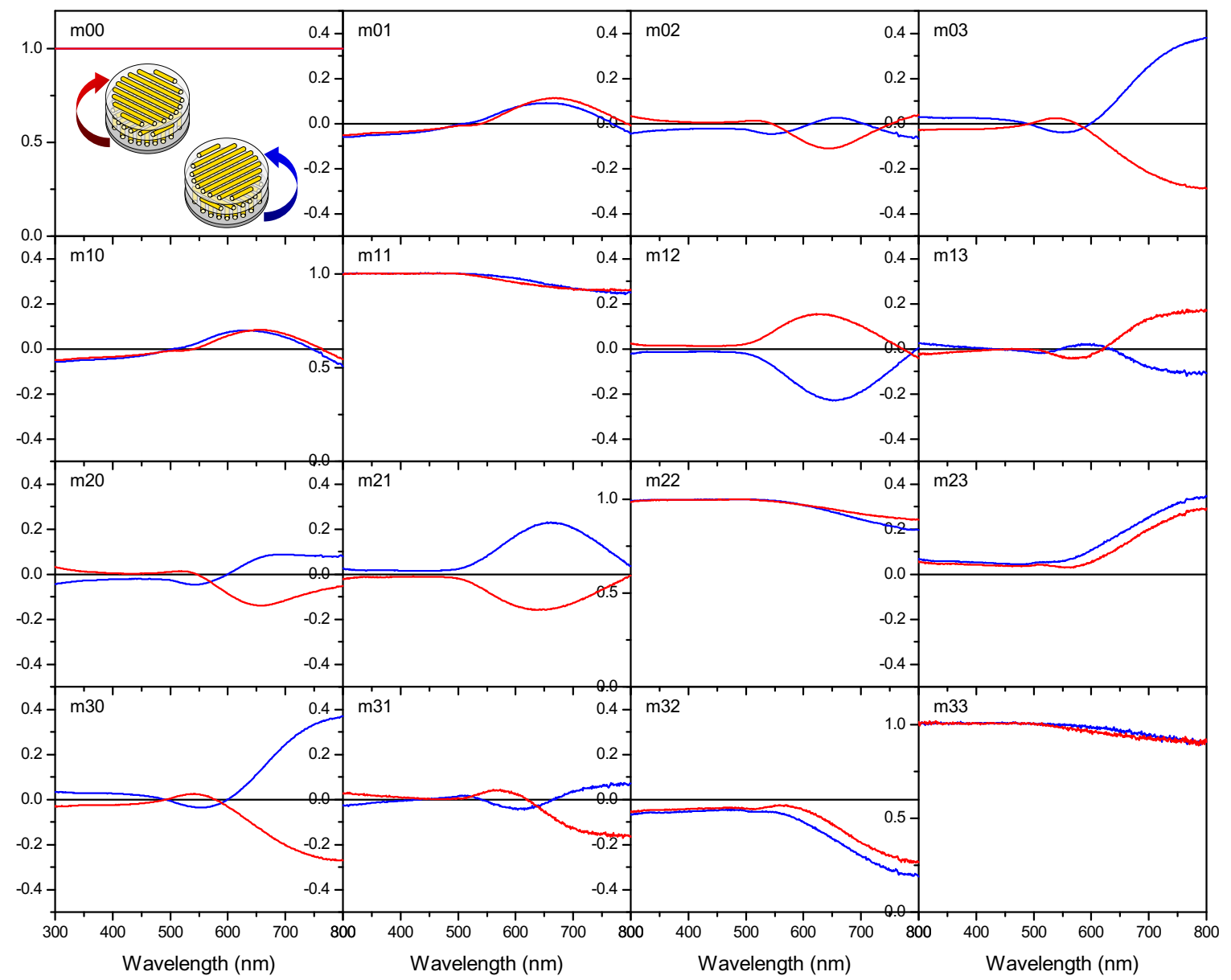

Figure S9: Mueller Matrix measured for a left- and right-handed 3-layer AuNR chiral metasurface.

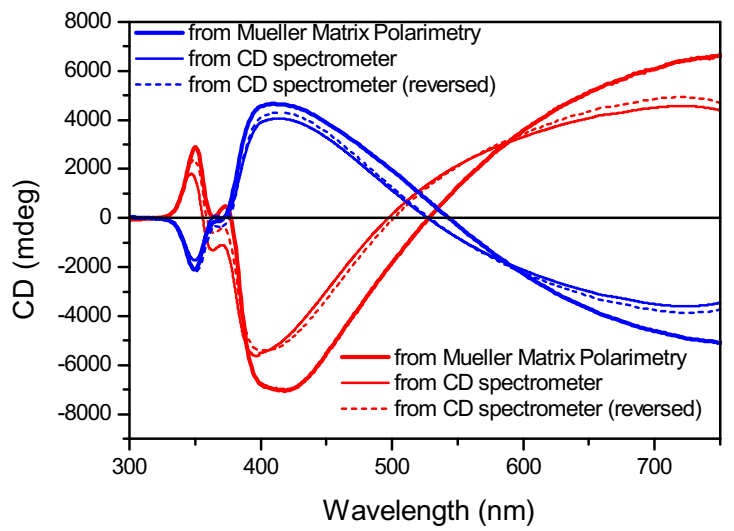

Figure S10: CD measured by Mueller Matrix Polarimetry compared to the CD measured from the commercial Jasco CD spectrometer. For the latter, the sample was first placed so that the substrate is facing the incoming light beam, and then reversed so that the nanowire multilayer is facing the light beam. 


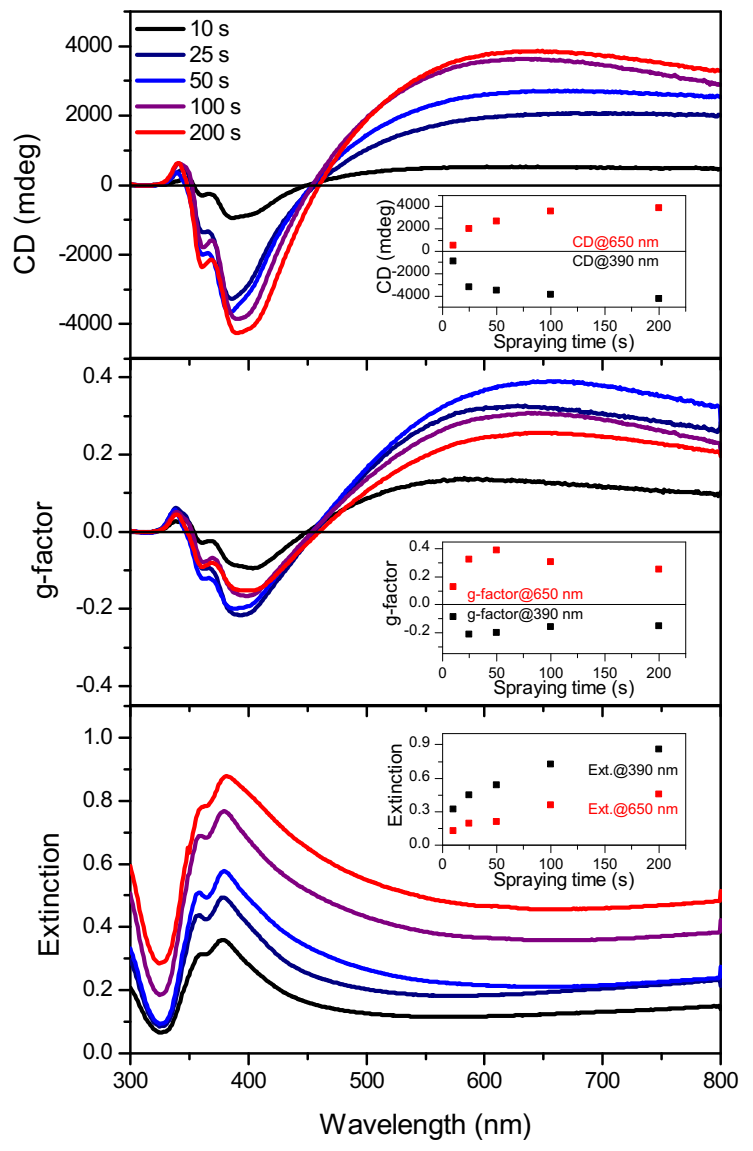

Figure S11: measured CD, g-factor and Extinction spectra of chiral AgNW 3-layer metasurfaces as function of the spraying time of each layer. The inset of each panel displays the value of the CD, g-factor and extinction at 650 $\mathrm{nm}$ and $390 \mathrm{~nm}$ as function of the spraying time. 


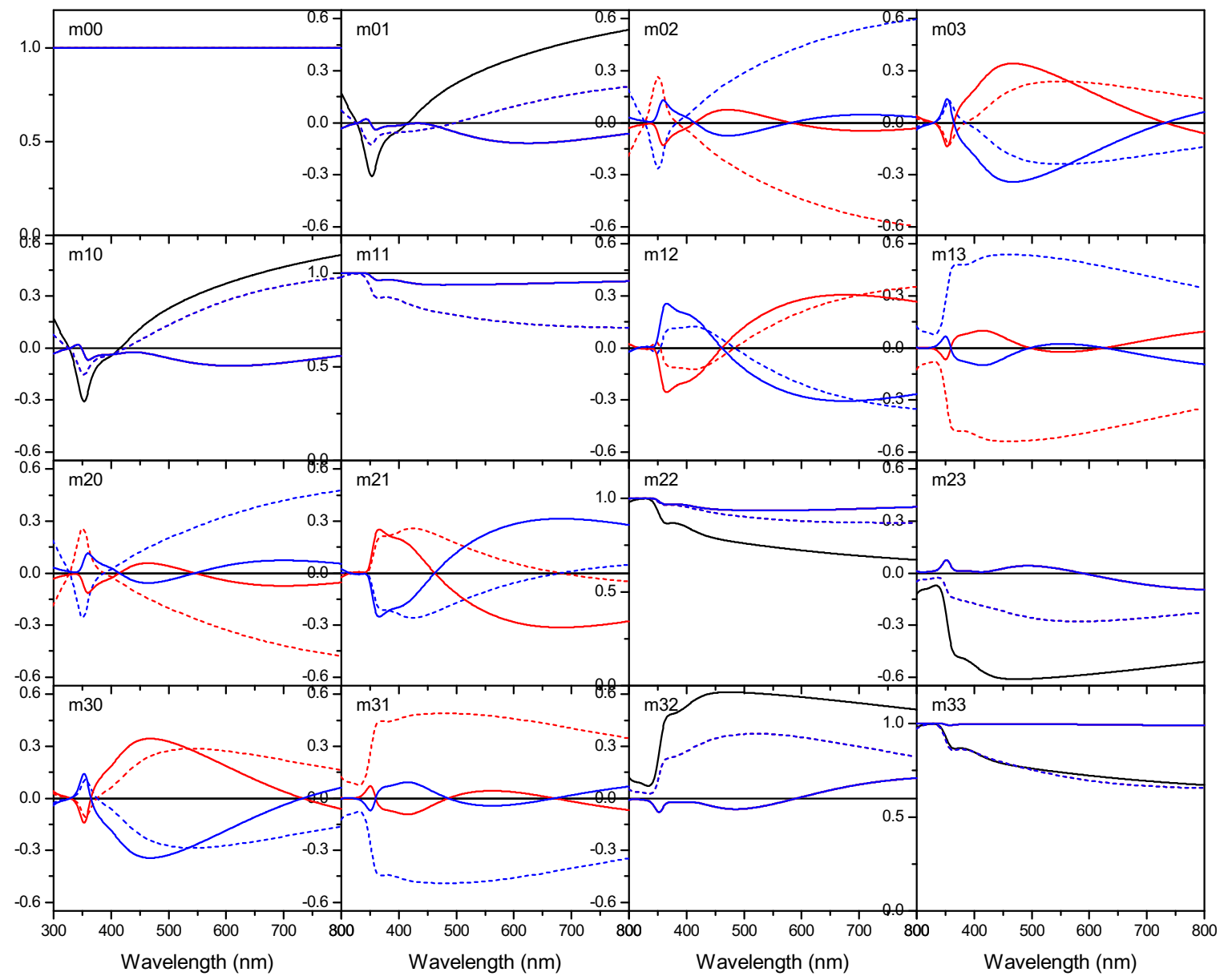

Figure S12: modeled Mueller Matrix for a 1, 2 and 3-layer metasurface of AgNWs. The spectra of the monolayer sample are in black, the spectra for the 2-layer thin films are the dashed lines while the spectra of the 3-layer films are in full lines (red for left-handed samples and blue for right-handed samples) The simulation is based on the complex ordinary and extraordinary effective dielectric function of a monolayer measured by MMP, which is used in a transfer matrix simulation of the multilayer nanostructures. 


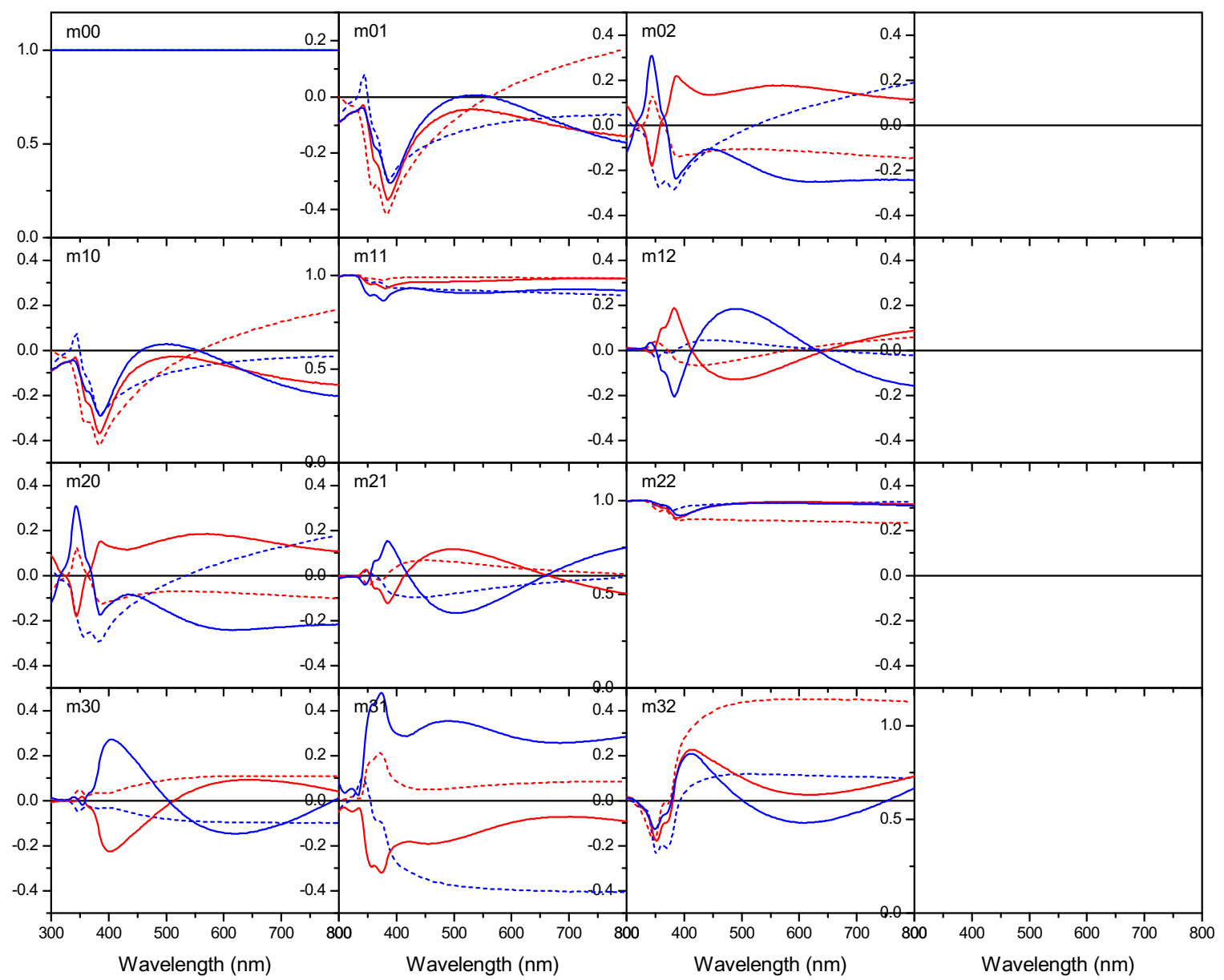

Figure S13: measured Mueller Matrix for a left- and right-handed 3-layer AgNW chiral metasurface. The AgNWs used here are commercially available. The spectra of the monolayer sample are in black, the spectra for the 2layer thin films are the dashed lines while the spectra of the 3-layer films are in full lines (red for left-handed samples and blue for right-handed samples)

\section{Supplementary references}

1. Schubert, M., Polarization-Dependent Optical Parameters of Arbitrarily Anisotropic Homogeneous Layered Systems. Phys. Rev. B 1996, 53, 4265-4274.

2. Pérez, J. J. G.; Ossikovski, R., Polarized Light and the Mueller Matrix Approach. CRC Press, Taylor \& Francis Group: Boca Raton (USA), 2016; p 405. 